\title{
Log Néron models over surfaces, II
}

\author{
Chikara NAKAYAMA
}

(Received May 8, 2013; Revised February 25, 2014)

\begin{abstract}
We prove that an admissible normal function over a surface and the zero section simultaneously extend to sections of a log Néron model. This gives a new proof of the surface base case of the algebraicity of zero loci of admissible normal functions.

Key words: Hodge theory, log geometry, Néron model, admissible normal function.
\end{abstract}

\section{Introduction}

In [10], we prove that, for a given admissible normal function $\nu$ ([11]) over a surface, there is a log Néron model which "graphs" it, in the sense that $\nu$ extends to a section of the model.

A next natural problem is to construct a model which graphs two given admissible normal functions simultaneously.

In this paper, we study the special case of this problem where one of the two functions is the zero section. As an application, we give an alternative, simple proof of the case of the surface base of the theorem by P. Brosnan and G. Pearlstein [1] on the algebraicity of zero loci of admissible normal functions (independently proved by C. Schnell [13] and by K. Kato, C. Nakayama, and S. Usui [6]).

In Section 1, we state the main result. From Section 2 to Section 4, we prove it. The proof goes roughly as follows. Let $\nu$ be an admissible normal function over a surface. Let $\sigma$ be the local monodromy cone associated to $\nu$. Let $\sigma_{0}^{\prime}$ be the cone associated to the zero section. The problem is to prove that there is a finite subdivision of $\sigma$ for each member $\tau$ of which, the translations of $\tau$ and those of the zero section $\tau_{0}^{\prime}$ by $G_{\mathbf{Z}}$ make a single weak fan. Here $G_{\mathbf{Z}}$ is the group of automorphisms of the lattice and "weak fan" is a relaxed concept of fan, which admits some overlappings of cones. The proof of the existence of the subdivision is similar to that of [10]. A key ingredient is a property of polarized nilpotent orbits of two variables, which was proved in [10]. 
In the last section (Section 5), as an application, we give an alternative, simple proof of the algebraicity of the zero locus of an admissible normal function in the surface case. We remark that, if one would generalize the construction in this paper to the case of the base of any dimension, it would give an alternative proof of the algebraicity over the general base.

As we explained in the introduction in [10], there are two ways to formulate log Néron models, i.e., an absolute formulation and a relative formulation (cf. [3] and [7, Section 5] respectively). We worked with the absolute formulation in [10]. Since we adopt the relative formulation in this paper, the result in [10] is not contained in the result in this paper.

Notation and Terminology. All combinatorial notions are the rational ones, i.e., are considered over $\mathbf{Q}$. For example, a polyhedral cone is a finitely generated, cancellative $\mathbf{Q}_{\geq 0}$-monoid. A fan in a $\mathbf{Q}$-vector space $V$ is a set $\Sigma$ of strictly convex polyhedral cones in $V$ satisfying: (1) A face of a member of $\Sigma$ also belongs to $\Sigma$. (2) For $\sigma, \sigma^{\prime} \in \Sigma$, the intersection $\sigma \cap \sigma^{\prime}$ is a face of $\sigma$. A finite subdivision of a polyhedral cone $\sigma$ in $V$ is a finite fan $\Sigma$ in $V$ whose support coincides with $\sigma$.

Let $N: V \rightarrow V^{\prime}$ be a map of sets. For a subset $A$ of $V$ and a subset $A^{\prime}$ of $V^{\prime}$, we write $N A$ for $N(A)$ and $N^{-1} A^{\prime}$ for $N^{-1}\left(A^{\prime}\right)$. For example, for maps $N_{1}, N_{2}: V \rightarrow V^{\prime}$, the symbol $\left(N_{2} N_{1}^{-1}\right)^{2} A^{\prime}$ means $N_{2}\left(N_{1}^{-1}\left(N_{2}\left(N_{1}^{-1}\left(A^{\prime}\right)\right)\right)\right)$.

\section{Main results}

1.1 First we review the definition of weak fans. As is explained in [7, Section 2] and in ibid. Section 5 respectively, there are an absolute formulation and a relative formulation of weak fans. In this paper, we use weak fans in the relative setting, that is, the ones in [7, Section 5]. Thus the following definition is the same as the one in [7, Section 5] except that we work over $\mathbf{Q}$, which does not yield any difference in essence.

1.2 We fix a free $\mathbf{Z}$-module $H_{\mathbf{Z}}^{\prime}$ of finite rank and define $H_{\mathbf{Z}}:=H_{\mathbf{Z}}^{\prime} \oplus \mathbf{Z}$. Let $W$ be the increasing filtration on $H_{\mathbf{Q}}:=H_{\mathbf{Z}} \otimes \mathbf{Q}$ characterized by $\operatorname{gr}_{-1}^{W}\left(H_{\mathbf{Q}}\right)=H_{\mathbf{Q}}^{\prime}$ and $\operatorname{gr}_{0}^{W}\left(H_{\mathbf{Q}}\right)=\mathbf{Q}$. Let $\langle,\rangle_{-1}: H_{\mathbf{Z}}^{\prime} \times H_{\mathbf{Z}}^{\prime} \rightarrow \mathbf{Q}$ be a non-degenerate anti-symmetric pairing. Let $\langle,\rangle_{0}$ be the pairing $\mathbf{Z} \times \mathbf{Z} \rightarrow$ $\mathbf{Q} ;(a, b) \mapsto a b$. Let $\left(h^{p, q}\right)_{p, q}$ are non-negative integers given for any $p, q \in \mathbf{Z}$ satisfying the following conditions (1)-(4).

(1) $h^{p, q}=0$ unless $p+q=-1$ or $p=q=0$. 
(2) $h^{0,0}=1$.

(3) $\sum_{p+q=-1} h^{p, q}=\operatorname{rank}_{\mathbf{Z}} H_{\mathbf{Z}}^{\prime}$.

(4) $h^{p, q}=h^{q, p}$ for any $p, q$.

Let $D$ be the associated classifying space of mixed Hodge structures with polarized graded quotients, introduced by S. Usui [14].

1.3 For $A=\mathbf{Z}, \mathbf{Q}$, let $G_{A}^{\prime}$ be the group of the $A$-automorphisms of $\left(H_{A}^{\prime},\langle,\rangle_{-1}\right)$. Let $G_{A}$ be the group of the $A$-automorphisms of $\left(H_{A}, W \cap\right.$ $\left.H_{A},\langle,\rangle_{-1},\langle,\rangle_{0}\right)$.

Let $\mathfrak{g}_{\mathbf{Q}}^{\prime}$ be the Lie algebra associated to $G_{\mathbf{Q}}^{\prime}$ and $\mathfrak{g}_{\mathbf{Q}}$ the Lie algebra associated to $G_{\mathbf{Q}}$.

1.4 Let $P$ be a sharp fs monoid. Let $\sigma^{\prime}:=\operatorname{Hom}\left(P, \mathbf{Q}_{\geq 0}^{\text {add }}\right)$ be the dual cone, where $\mathbf{Q}_{\geq 0}^{\text {add }}$ is the monoid of non-negative rational numbers with respect to addition.

Let $\Gamma^{\prime}$ be the abelian group $\operatorname{Hom}\left(P^{\mathrm{gp}}, \mathbf{Z}\right)$. Assume that a homomorphism

$$
a: \Gamma^{\prime} \rightarrow G_{\mathbf{Z}, u}^{\prime}
$$

of groups is given, where $G_{\mathbf{Z}, u}^{\prime}$ is the unipotent part of $G_{\mathbf{Z}}^{\prime}$. Let

$$
\sigma^{\prime} \rightarrow \mathfrak{g}_{\mathbf{Q}}^{\prime}
$$

be the homomorphism of monoids induced by the logarithm of $a$.

1.5 A nilpotent cone is a polyhedral cone $\sigma$ in the fiber product

$$
\sigma^{\prime} \times_{\mathfrak{g}_{\mathbf{Q}}^{\prime}} \mathfrak{g}_{\mathbf{Q}}
$$

whose image $\sigma_{\mathfrak{g}}$ in $\mathfrak{g}_{\mathbf{Q}}$ is a nilpotent cone in the absolute sense, i.e., a polyhedral cone consisting of mutually commutative nilpotent elements (cf. [10, $1.4])$.

We say that $\sigma$ is admissible if $\sigma_{\mathfrak{g}}$ is admissible.

Let $\sigma$ be a nilpotent cone and let $F$ be an element of the compact dual $\check{D}$ of $D$. We say that $(\sigma, F)$ generates a nilpotent orbit if $\left(\sigma_{\mathfrak{g}}, F\right)$ generates a nilpotent orbit in the absolute sense $([10,1.4])$.

We say that $\sigma$ is sharp if it is strictly convex, i.e., $\sigma \cap(-\sigma)=\{0\}$. 
1.6 Let $N^{\prime} \in \mathfrak{g}_{\mathbf{Q}}^{\prime}$. Let $e$ be the standard generator $1 \in \mathbf{Z}=\operatorname{gr}_{0}^{W}\left(H_{\mathbf{Z}}\right) \subset$ $H_{\mathbf{Z}}$. Then, there is a unique element $N_{0}^{\prime} \in \mathfrak{g}_{\mathbf{Q}}$ whose image in $\mathfrak{g}_{\mathbf{Q}}^{\prime}$ coincides with $N^{\prime}$ and which satisfies $N_{0}^{\prime}(e)=0$. Let

$$
j: \mathfrak{g}_{\mathbf{Q}}^{\prime} \hookrightarrow \mathfrak{g}_{\mathbf{Q}}
$$

be the injective homomorphism defined by $j\left(N^{\prime}\right)=N_{0}^{\prime}$.

Let $\tau^{\prime}$ be a polyhedral subcone of $\sigma^{\prime}$. Then, the 0 -lift of $\tau^{\prime}$, denoted by $\tau_{0}^{\prime}$, is the nilpotent cone in $\sigma^{\prime} \times_{\mathfrak{g}_{\mathbf{Q}}^{\prime}} \mathfrak{g}_{\mathbf{Q}}$ defined by

$$
\tau_{0}^{\prime}=\left\{\left(x, j\left(N_{x}^{\prime}\right)\right) \mid x \in \tau^{\prime}\right\}
$$

where $N_{x}^{\prime}$ is the image of $x$ by $\sigma^{\prime} \rightarrow \mathfrak{g}_{\mathbf{Q}}^{\prime}$.

1.7 A weak fan $\Sigma$ in $\sigma^{\prime} \times_{\mathfrak{g}_{\mathbf{Q}}^{\prime}} \mathfrak{g}_{\mathbf{Q}}$ is a non-empty set of sharp nilpotent cones satisfying the following conditions (1) and (2).

(1) Any face of an element of $\Sigma$ also belongs to $\Sigma$.

(2) Let $\sigma_{1}, \sigma_{2} \in \Sigma$. Assume that they have a common interior point. Assume also that there is an $F \in \check{D}$ such that $\left(\sigma_{1}, F\right)$ and $\left(\sigma_{2}, F\right)$ generate nilpotent orbits. Then $\sigma_{1}=\sigma_{2}$.

A fan in $\sigma^{\prime} \times_{\mathfrak{g}_{\mathbf{Q}}^{\prime}} \mathfrak{g}_{\mathbf{Q}}$ is defined, as usual, by replacing (2) with the condition that for $\sigma_{1}, \sigma_{2} \in \Sigma$, the intersection $\sigma_{1} \cap \sigma_{2}$ is a face of $\sigma_{1}$.

Any fan is a weak fan $([7,5.1 .6]$, cf. $[5,1.7])$, but the converse is not valid in general.

1.8 Next we review log Néron models and their variants.

Let $\Gamma:=\Gamma^{\prime} \times_{G_{\mathbf{Z}}^{\prime}} G_{\mathbf{Z}}$, which acts on $\sigma^{\prime} \times_{\mathfrak{g}_{\mathbf{Q}}^{\prime}} \mathfrak{g}_{\mathbf{Q}}$ via

$$
\operatorname{Ad}(\gamma)((x, y))=\left(x, \operatorname{Ad}\left(\gamma_{G}\right) y\right) \quad\left(\gamma \in \Gamma,(x, y) \in \sigma^{\prime} \times_{\mathfrak{g}_{\mathbf{Q}}^{\prime}} \mathfrak{g}_{\mathbf{Q}}\right)
$$

Here $\gamma_{G}$ is the image of $\gamma$ in $G_{\mathbf{Z}}$.

Let $\Sigma$ be a weak fan which is strongly compatible with $\Gamma([7,5.1 .8])$.

Let $\mathcal{B}(\log )$ be the category of the spaces which are locally isomorphic to strong subspaces of fs log analytic spaces $([9,3.2 .4])$.

Let $S_{0}:=E_{\sigma^{\prime}}^{\prime}$ be the object in $\mathcal{B}(\log )$ which is defined in $[7,5.1 .1]$ from the data $\left(H_{\mathbf{Z}}, W,\langle,\rangle_{-1},\langle,\rangle_{0},\left(h^{p, q}\right)_{p, q}\right)$ in 1.2 and $P, a$ in 1.4 .

There is a canonical polarized log Hodge structure (PLH) of weight -1 
on $S_{0}$.

Let $\varphi: S \rightarrow S_{0}$ be a strict morphism in $\mathcal{B}(\log )$, where a morphism is said to be strict if the pullback of the $\log$ structure on the target space is naturally isomorphic to that on the source space.

Let $H^{\prime}$ be a PLH of weight -1 on $S$, and assume that we are given an isomorphism $\iota$ of PLHs between $H^{\prime}$ and the pullback of the canonical PLH of weight -1 on $S_{0}$ by $\varphi$.

Let $D_{S, \Sigma}$ be the space of nilpotent orbits in the relative formulation ([7, 5.1.10]). The quotient $J_{S, \Sigma}:=\Gamma \backslash D_{S, \Sigma}$ is endowed with the structure of an object in $\mathcal{B}(\log )$.

Then, main results in [7, Section 5] say that $J_{S, \Sigma}$ is a nice space in various senses; for instance, by Theorem 5.2.8 of [7], the space $J_{S, \Sigma}$ is Hausdorff if $S$ is Hausdorff.

1.9 By another main theorem (Theorem 5.3.3) of [7], the space $J_{S, \Sigma}$ represents the following functor.

Let $\mu^{\prime}$ be the $\Gamma^{\prime}$-level structure on $H^{\prime}$ via the isomorphism $\iota$. Then, the functor represented by $J_{S, \Sigma}$ associates to $T \in \mathcal{B}(\log ) / S$ the set of isomorphism classes of a log mixed Hodge structure (LMH) $H$ on $T$ satisfying the following conditions (1) and (2).

(1) $\operatorname{gr}_{w}^{W}(H)$ is identified with the pullback of $H^{\prime}, \mathbf{Z}$ (the Tate's Hodge structure), and 0 if $w=-1, w=0$, and $w \neq 0,-1$, respectively.

(2) Under the identification in (1), for any $t \in T^{\log }$, if $\tilde{\mu}_{t}: H_{t} \stackrel{\sim}{\rightarrow} H_{\mathbf{Z}}\left(H_{t}\right.$ here denotes the stalk at $t$ of the lattice of $H$ ) is any isomorphism preserving the weight filtration whose $\operatorname{gr}_{-1}^{W}$ belongs to $\mu^{\prime}$ and whose $\operatorname{gr}_{0}^{W}$ is the identity, then there exists a cone $\sigma \in \Sigma$ such that $\sigma$ contains the image of the map $\pi_{1}^{+}\left(\tau^{-1} \tau(t)\right) \rightarrow \sigma^{\prime} \times_{\mathfrak{g}_{\mathbf{Q}}^{\prime}} \mathfrak{g}_{\mathbf{Q}}$ whose first component is induced by $T \rightarrow S \stackrel{\varphi}{\rightarrow} S_{0}=E_{\sigma^{\prime}}^{\prime}$ and whose second component is induced by $\tilde{\mu}_{t}$ (cf. $[7,2.6 .2])$, and such that $\left(\sigma, \tilde{\mu}_{t}\left(\mathbf{C} \otimes_{\mathcal{O}_{T, t}^{\log }} F_{t}\right)\right)$ generates a nilpotent orbit.

Here $\pi_{1}^{+}\left(\tau^{-1} \tau(t)\right):=\operatorname{Hom}\left(\left(M_{T} / \mathcal{O}_{T}^{\times}\right)_{\tau(t)}, \mathbf{N}\right) \subset \operatorname{Hom}\left(\left(M_{T} / \mathcal{O}_{T}^{\times}\right)_{\tau(t)}\right.$, $\mathbf{Z})=\pi_{1}\left(\tau^{-1} \tau(t)\right), F$ is the Hodge filtration of $H$, and $\mathbf{C} \otimes_{\mathcal{O}_{T, t}^{\log }} F_{t}$ is independent of the choice of a $\mathbf{C}$-algebra homomorphism $\mathcal{O}_{T, t}^{\log } \rightarrow \mathbf{C}$. (We also use the standard notation $\tau$ for the canonical projection $X^{\log } \rightarrow X$ for an fs log analytic space $X$.) 
We have an embedding

$$
\operatorname{Mor}\left(-, J_{S, \Sigma}\right) \subset \mathcal{E} x t^{1}\left(\mathbf{Z}, H^{\prime}\right)
$$

of functors from the category $\mathcal{B}(\log ) / S$ to the category of sets. Here $\mathcal{E} x t^{1}$ is the sheaf $T \mapsto \operatorname{Ext}_{T}^{1}\left(\mathbf{Z},\left.H^{\prime}\right|_{T}\right)$ in the category of log mixed Hodge structures on $T$. The image of this embedding consists of $H$ satisfying the following (3).

(3) For any $t \in T^{\log }$, if $\tilde{\mu}_{t}^{\prime}: H_{t}^{\prime} \stackrel{\sim}{\rightarrow} H_{\mathbf{Z}}^{\prime}\left(H_{t}^{\prime}\right.$ here denotes the stalk at $t$ of the lattice of $H^{\prime}$ ) is a representative of the germ of $\mu^{\prime}$ at $t$, then there exists a cone $\sigma \in \Sigma$ such that $\sigma$ contains the image of the map $\pi_{1}^{+}\left(\tau^{-1} \tau(t)\right) \rightarrow \sigma^{\prime} \times_{\mathfrak{g}_{\mathbf{Q}}^{\prime}} \mathfrak{g}_{\mathbf{Q}}$ induced by $\varphi$ and $\tilde{\mu}_{t}^{\prime} \oplus \mathrm{id}$, and such that $\left(\sigma,\left(\tilde{\mu}_{t}^{\prime} \oplus \mathrm{id}\right)\left(\mathbf{C} \otimes_{\mathcal{O}_{T} \log , t} F_{t}\right)\right)$ generates a nilpotent orbit.

Now we proceed to state the results.

We first state the results in 1-dimensional base case for the readers' convenience.

Proposition 1.10 Let $P, \sigma^{\prime}, \Gamma^{\prime}$, and a be as in 1.4. Let $\Gamma$ be as in 1.8. Assume that $\operatorname{dim} \sigma^{\prime} \leq 1$. Let $\sigma$ be a nilpotent cone in $\sigma^{\prime} \times_{\mathfrak{g}_{\mathbf{Q}}^{\prime}} \mathfrak{g}_{\mathbf{Q}}$ such that $\sigma \rightarrow \sigma^{\prime}$ is bijective. Then, the set of the translations $\operatorname{Ad}(\gamma)(\sigma)$ of $\sigma$ and the translations $\operatorname{Ad}(\gamma)\left(\sigma_{0}^{\prime}\right)$ of the 0-lift $\sigma_{0}^{\prime}(1.6)$ by all the elements $\gamma$ of $\Gamma$ together with the trivial cone $\{0\}$ form a fan.

This is just because the dimensions of all these cones are 0 or 1 .

Corollary 1.11 Let $S$ be an object of $\mathcal{B}(\log )$. Assume that the log rank of $S$ is equal to or less than 1 , that is, the monoid $\left(M_{S} / \mathcal{O}_{S}^{\times}\right)_{s}$ is isomorphic to either $\mathbf{N}$ or $\{0\}$ for any $s \in S$. Let $H^{\prime}$ be a PLH of weight -1 on $S$. Let $\nu$ be an element of $\operatorname{Ext}^{1}\left(\mathbf{Z}, H^{\prime}\right)$ and $\nu_{0} \in \operatorname{Ext}^{1}\left(\mathbf{Z}, H^{\prime}\right)$ the trivial extension. Then, locally on $S$, there are Hodge data as in 1.2, $P$ and a as in 1.4, a fan $\Sigma$ (being strongly compatible with $\Gamma$ ), and $\varphi$ and $\iota$ as in 1.8 such that $J_{S, \Sigma}$ graphs $\nu$ and $\nu_{0}$ simultaneously, which means that both $\nu$ and $\nu_{0}$ belong to $\operatorname{Mor}\left(S, J_{S, \Sigma}\right) \subset \operatorname{Ext}^{1}\left(\mathbf{Z}, H^{\prime}\right)$.

We call such $J_{S, \Sigma}$ a log Néron model for $\nu$ and $\nu_{0}$.

We remark that in this case where the log rank is equal to or less than 1, the Néron model in the sense of [7] exists as a best model and satisfies the same (or even stronger) conclusion. See [7, Corollary 6.1.6]. But we give 
here a proof of Corollary 1.11 based on Proposition 1.10 which is helpful to understand the 2-dimensional correspondent Corollary 1.14 below.

Proof. Let $s$ be a point of $S$ and we work around $s$. Let $P:=\left(M_{S} / \mathcal{O}_{S}^{\times}\right)_{s}$, and $\sigma^{\prime}:=\operatorname{Hom}\left(P, \mathbf{Q}_{\geq 0}^{\text {add }}\right)$. By localizing $S$, we may assume that there is a chart $P \rightarrow \Gamma\left(S, M_{S}\right)$ such that the induced $P \rightarrow\left(M_{S} / \mathcal{O}_{S}^{\times}\right)_{s}$ is the identity.

As is explained in $[7,5.1 .2]$, locally on $S$, we can find Hodge data as in 1.2, a homomorphism $a$ as in 1.4, and data $\varphi$ and $\iota$ as in 1.8.

Let $H$ be the LMH corresponding to $\nu$. Let $t \in S^{\log }$ such that $s=\tau(t)$. Let $\tilde{\mu}_{t}^{\prime}$ be as in $1.9(3)$. Then, via $\tilde{\mu}_{t}^{\prime} \oplus \mathrm{id}: H_{t} \stackrel{\sim}{\rightarrow} H_{\mathbf{Z}}$, the monoid $\pi_{1}^{+}\left(\tau^{-1}(s)\right)$ acts on $H_{\mathbf{Z}}$.

Let $\sigma$ be the local monodromy cone of $H$ at $s$, that is, the cone in $\sigma^{\prime} \times_{\mathfrak{g}_{\mathbf{Q}}^{\prime}}$ $\mathfrak{g}_{\mathbf{Q}}$ generated by the elements of the form $(x, y)$, where $y$ is the logarithm of the action of $x \in \pi_{1}^{+}\left(\tau^{-1}(s)\right)=\operatorname{Hom}(P, \mathbf{N}) \subset \sigma^{\prime}$. It is admissible, and is determined up to the translation by an element of $\Gamma$. Further, $\operatorname{dim} \sigma \leq 1$ and the canonical map $\sigma \rightarrow \sigma^{\prime}$ is bijective.

Now we apply Proposition 1.10 which gives the fan $\Sigma$ as in the statement of Proposition 1.10. It is clearly strongly compatible with $\Gamma$.

We will show that $J_{S, \Sigma}$ graphs $\nu$ and $\nu_{0}$. For this, it suffices to verify the condition (3) in 1.9 for $\nu$ and for $\nu_{0}$. We can take the local monodromy cone $\sigma$ for $\nu$ and the 0 -lift $\sigma_{0}^{\prime}(1.6)$ for $\nu_{0}$ as the desired cone in the condition (3) in 1.9 .

Corollary 1.12 Let $S$ be a complex analytic manifold endowed with the log structure defined by a smooth divisor $Z$. Then for any normal function $\nu$ on $U:=S-Z$ which is admissible with respect to $S$ ([11]), locally on $S$, there are Hodge data as in 1.2, $P$ and a as in 1.4, a fan $\Sigma$ (being strongly compatible with $\Gamma$ ), and $\varphi$ and $\iota$ as in 1.8 such that $\nu$ and the trivial normal function $\nu_{0}$ extend to sections of $J_{S, \Sigma}$.

Proof. Let $H^{\prime}$ be the PHS corresponding to $\operatorname{gr}_{-1}^{W}(\nu)$. By the nilpotent orbit theorem of Schmid ([12]) reformulated as in [2, Proposition 2.5] or $[9,2.5 .13$-Theorem 2.5.14], it extends uniquely to a PLH over $S$, which we still denote by $H^{\prime}$. A normal function on $U$ is nothing but an element of $\operatorname{Ext}_{U}^{1}\left(\mathbf{Z}, H^{\prime}\right)$. By the admissibility, it extends to an element of $\operatorname{Ext}_{S}^{1}\left(\mathbf{Z}, H^{\prime}\right)$. Hence this corollary is reduced to the previous one. (Note that the log rank is equal to or less than 1 for $Z$ is smooth.) 
Again, the Néron model in the sense of [7] satisfies the same conclusion. See [7, Corollary 6.1.8].

We proceed to the surface base case. The next is the main theorem in this paper, which is proved in Section 4 after the necessary preparations.

Theorem 1.13 Let $P, \sigma^{\prime}, \Gamma^{\prime}$, and $a$ be as in 1.4. Let $\Gamma$ be as in 1.8. Assume that $\operatorname{dim} \sigma^{\prime}=2$. Let $\sigma$ be an admissible nilpotent cone in $\sigma^{\prime} \times_{\mathfrak{g}_{\mathbf{Q}}^{\prime}} \mathfrak{g}_{\mathbf{Q}}$ such that $\sigma \rightarrow \sigma^{\prime}$ is bijective. Then, there exists a finite subdivision of $\sigma$ for each member $\tau$ of which, the set $\Sigma:=\{\operatorname{Ad}(\gamma)(v) \mid \gamma \in \Gamma$, $v$ is a face of $\tau$ or a face of $\left.\tau_{0}^{\prime}\right\}$ is a weak fan. Here $\tau_{0}^{\prime}$ is the 0-lift (1.6) of the image of $\tau$ in $\mathfrak{g}_{\mathbf{Q}}^{\prime}$.

We expect that the conclusion in Theorem 1.13 would hold without the assumption $\operatorname{dim} \sigma^{\prime}=2$. Another problem is to generalize Theorem 1.13 to the case where $\sigma \rightarrow \sigma^{\prime}$ is only surjective.

Corollary 1.14 Let $S$ be an object of $\mathcal{B}(\log )$. Assume that the log rank of $S$ is equal to or less than 2 , that is, $\operatorname{rank}_{\mathbf{Z}}\left(\left(M_{S}^{\mathrm{gp}} / \mathcal{O}_{S}^{\times}\right)_{s}\right) \leq 2$ for any $s \in S$. Let $H^{\prime}$ be a PLH of weight -1 on $S$. Let $\nu$ be an element of $\operatorname{Ext}_{S}^{1}\left(\mathbf{Z}, H^{\prime}\right)$. Then, locally on $S$, there is a log modification $([9,3.6]) S^{\prime} \rightarrow S$ and, locally on $S^{\prime}$, there are Hodge data as in 1.2, $P$ and a as in 1.4, a fan $\Sigma$ (being strongly compatible with $\Gamma$ ), and $\varphi$ and $\iota$ as in 1.8 such that $J_{S^{\prime}, \Sigma}$ graphs $\left.\nu\right|_{S^{\prime}} \in \operatorname{Ext}_{S^{\prime}}^{1}\left(\mathbf{Z},\left.H^{\prime}\right|_{S^{\prime}}\right)$ and the trivial extension $\nu_{0} \in \operatorname{Ext}_{S^{\prime}}^{1}\left(\mathbf{Z},\left.H^{\prime}\right|_{S^{\prime}}\right)$ simultaneously.

We call such $J_{S, \Sigma}$ a log Néron model for $\nu$ and $\nu_{0}$.

Proof. The proof is parallel to that of Corollary 1.11.

Let $s$ be a point of $S, P:=\left(M_{S} / \mathcal{O}_{S}^{\times}\right)_{s}$, and $\sigma^{\prime}:=\operatorname{Hom}\left(P, \mathbf{Q}_{\geq 0}^{\text {add }}\right)$. Similarly as in Corollary 1.11, we work around $s$ and may assume that there are a chart $P \rightarrow \Gamma\left(S, M_{S}\right)$ such that the induced $P \rightarrow\left(M_{S} / \mathcal{O}_{S}^{\times}\right)_{s}$ is the identity, the Hodge data in 1.2, and $a$ in 1.4, and $\varphi$ and $\iota$ in 1.8.

Let $H$ be the LMH corresponding to $\nu$. Let $t \in S^{\log }$ with $s=\tau(t)$. The monoid $\pi_{1}^{+}\left(\tau^{-1}(s)\right)$ acts on $H_{\mathbf{Z}}$ via $\tilde{\mu}_{t}^{\prime} \oplus \mathrm{id}$, where $\tilde{\mu}_{t}^{\prime}$ is as in 1.9 (3).

Let $\sigma \subset \sigma^{\prime} \times \mathfrak{g}_{\mathbf{Q}}^{\prime} \mathfrak{g}_{\mathbf{Q}}$ be the local monodromy cone of $H$ at $s$. Then, $\operatorname{dim} \sigma \leq 2$ and the canonical map $\sigma \rightarrow \sigma^{\prime}$ is bijective.

Further, for any $t^{\prime} \in S^{\log }, \pi_{1}^{+}\left(\tau^{-1} \tau\left(t^{\prime}\right)\right)$ is regarded as a face of $\pi_{1}^{+}\left(\tau^{-1}(s)\right)$ (because $\left(M_{S} / \mathcal{O}_{S}^{\times}\right)_{\tau\left(t^{\prime}\right)}$ is regarded as a quotient of $\left.\left(M_{S} / \mathcal{O}_{S}^{\times}\right)_{s}\right)$, and the action of $\pi_{1}^{+}\left(\tau^{-1} \tau\left(t^{\prime}\right)\right)$ on $H_{\mathbf{Z}}$ factors through the action of 
$\pi_{1}^{+}\left(\tau^{-1}(s)\right)$ on $H_{\mathbf{Z}}$ modulo the translation by an element of $\Gamma$.

Now, take a log modification $S^{\prime}$ of $S$ according to the finite subdivision of $\sigma^{\prime}$ induced by that of $\sigma$ which Theorem 1.13 gives. Let $\tau$ be as in Theorem 1.13. Then we have a weak fan $\Sigma$ in Theorem 1.13, which is strongly compatible with $\Gamma$. By localizing $S^{\prime}$, we may assume that $\Sigma$ contains the set of all local monodromy cones of $\left.H\right|_{S^{\prime}}$.

Then, $J_{S^{\prime}, \Sigma}$ graphs $\left.\nu\right|_{S^{\prime}}$ and $\nu_{0}$. To see it, at each point of $\left(S^{\prime}\right)^{\log \text {, we }}$ can take the local monodromy cone of $\left.\nu\right|_{S^{\prime}}$ (which is a face of $\tau$ ) to verify the condition (3) in 1.9 for $\nu$, and the local monodromy cone of $\nu_{0}$ (which is a face of the 0 -lift $\tau_{0}^{\prime}$ ) for $\nu_{0}$.

Corollary 1.15 Let $S$ be an fs log analytic space which is log smooth over C $([9,2.1 .11])$. Assume that the log rank of $S$ is equal to or less than 2 . Let $U$ be the open subspace of $S$ where the log structure is trivial. Then for any normal function $\nu$ on $U$ which is admissible with respect to $S$ ([11]), locally on $S$, there is a log modification $S^{\prime} \rightarrow S$ and, locally on $S^{\prime}$, there are Hodge data as in 1.2, $P$ and $a$ as in 1.4, a fan $\Sigma$ (being strongly compatible with $\Gamma)$, and $\varphi$ and $\iota$ as in 1.8 such that the morphism $U \stackrel{\nu}{\rightarrow} J_{S^{\prime}, \Sigma}$ and the trivial normal function $\nu_{0}$ extend to morphisms $S^{\prime} \rightarrow J_{S^{\prime}, \Sigma}$. (Note that, by the definition of a log modification, the open subspace $U$ of $S$ can be regarded as an open subspace of $S^{\prime}$.)

Proof. Similarly to Corollary 1.12, this is reduced to the previous corollary.

Remark 1.16 The example in $[13,6.3]$ shows that a log modification $S^{\prime} \rightarrow$ $S$ in the statement of Corollary 1.15 is indeed necessary. More precisely, let $U=\left(\Delta^{*}\right)^{2}$, and $S=\Delta^{2}$ endowed with the log structure defined by $S-U$. Consider the normal functions $\nu$ there. Then, $\nu$ and the trivial normal function $\nu_{0}$ cannot simultaneously extend over the origin to morphisms $S \rightarrow$ $J_{S, \Sigma}$ for any $\Sigma$. On the other hand, our proof shows that after log blowing up the origin of $S$, locally, they extend simultaneously.

\section{Subdivision of cones}

In this section, we prove a combinatorial lemma (Lemma 2.3), which will be used in the proof of the main theorem (Theorem 1.13).

2.1 Let $H$ be a finite dimensional $\mathbf{Q}$-vector space. Let 


$$
X=\mathbf{Q}_{\geq 0}^{2} \times H .
$$

Let $h_{1}, h_{2} \in H$. Let

$$
\sigma=\mathbf{Q}_{\geq 0}\left(1,0, h_{1}\right)+\mathbf{Q}_{\geq 0}\left(0,1, h_{2}\right) \subset X
$$

Let $L$ be a finitely generated free $\mathbf{Z}$-module. Let $N_{1}, N_{2} \in \operatorname{Hom}(L, H)$. Let $L$ act on $X$ by

$$
l:\left(a_{1}, a_{2}, h\right) \mapsto\left(a_{1}, a_{2}, h+\left(a_{1} N_{1}+a_{2} N_{2}\right)(l)\right) \quad(l \in L) .
$$

Note that, in applying the results in this section to the main theorem, we take $H_{\mathbf{Q}}^{\prime}$ in the main theorem as the $\mathbf{Q}$-vector space $H$ here.

We introduce the following notation. For a rational number $\varepsilon$ with $0 \leq \varepsilon \leq 1$, let

$$
H_{1+\varepsilon}=\mathbf{Q}_{\geq 0}(1-\varepsilon, \varepsilon) \times H .
$$

In particular,

$$
\begin{aligned}
& H_{1}=\mathbf{Q}_{\geq 0} \times\{0\} \times H, \text { and } \\
& H_{2}=\{0\} \times \mathbf{Q}_{\geq 0} \times H .
\end{aligned}
$$

Lemma 2.2 Let the notation and the assumption be as in 2.1. Then the intersection $\sigma \cap(\{(0,0)\} \times H)$ is trivial, that is, $\{(0,0,0)\}$.

Proof. Let $a_{1}\left(1,0, h_{1}\right)+a_{2}\left(0,1, h_{2}\right)=\left(a_{1}, a_{2}, a_{1} h_{1}+a_{2} h_{2}\right)\left(a_{1}, a_{2} \in \mathbf{Q}_{\geq 0}\right)$ be an element of $\sigma$. If it belongs to $\{(0,0)\} \times H$, both $a_{1}$ and $a_{2}$ must be zero, and this element should be $(0,0,0)$.

Lemma 2.3 Let the notation and the assumption be as in 2.1. Then we have the following.

(1) Assume that $N_{1}$ is injective. Then there is a positive $\varepsilon_{0} \leq 1$ such that any positive rational number $\varepsilon \leq \varepsilon_{0}$ satisfies the following condition: Let

$$
\sigma^{\prime}=\sigma \cap\left(H_{1}+H_{1+\varepsilon}\right)=\mathbf{Q}_{\geq 0}\left(1,0, h_{1}\right)+\mathbf{Q}_{\geq 0}\left(1-\varepsilon, \varepsilon,(1-\varepsilon) h_{1}+\varepsilon h_{2}\right) .
$$

Then, for any $l \in L$, we have either $(1,0,0) \in l\left(\sigma^{\prime}\right)$ or $l\left(\sigma^{\prime}\right) \cap\left(\mathbf{Q}_{\geq 0}^{2} \times\{0\}\right)=$ 
$\{(0,0,0)\}$.

(2) Assume that we are given an identification $L \otimes \mathbf{Q}=H$. We regard $N_{1}$ and $N_{2}$ as elements of $\operatorname{End}(H)$ via this identification. Let $J \subset H$ be a Q-subspace satisfying $N_{1}^{-1} N_{2} J \subset J$ (cf. Notation and Terminology). Then there is a positive $\varepsilon_{0} \leq 1$ such that any positive rational number $\varepsilon \leq \varepsilon_{0}$ satisfies the following condition: Let

$$
\sigma^{\prime}=\sigma \cap\left(H_{1}+H_{1+\varepsilon}\right)=\mathbf{Q}_{\geq 0}\left(1,0, h_{1}\right)+\mathbf{Q}_{\geq 0}\left(1-\varepsilon, \varepsilon,(1-\varepsilon) h_{1}+\varepsilon h_{2}\right) .
$$

Then, for any $l \in L$, we have either that $(1,0, h) \in l\left(\sigma^{\prime}\right)(h \in H)$ implies $h \in N_{1} J+N_{2} J$ or $l\left(\sigma^{\prime}\right) \cap\left(\mathbf{Q}_{\geq 0}^{2} \times\{0\}\right)=\{(0,0,0)\}$.

Proof. (1) Since $N_{1}$ is injective, there is a positive $\varepsilon_{0} \leq 1$ such that for any positive rational $\varepsilon \leq \varepsilon_{0}$, the operator $(1-\varepsilon) N_{1}+\varepsilon N_{2}$ is also injective. Hence, by replacing $N_{2}$ by $\left(1-\varepsilon_{0}\right) N_{1}+\varepsilon_{0} N_{2}$, and $X$ by $H_{1}+H_{1+\varepsilon_{0}}$, we may assume that $(1-\varepsilon) N_{1}+\varepsilon N_{2}$ is injective for any $0 \leq \varepsilon \leq 1$.

Next we show that we may further assume that $(1-\varepsilon) N_{1}+\varepsilon N_{2} \in$ $\operatorname{End}(H)$ is bijective for any $0 \leq \varepsilon \leq 1$. Take a free $\mathbf{Z}$-submodule $L^{\prime}$ of $H$ such that $N_{1} L \cap L^{\prime}=\{0\}$ and $N_{1} L \oplus L^{\prime}$ spans $H$ over $\mathbf{Q}$. Then, for any sufficiently small $\varepsilon$, the submodule $\left((1-\varepsilon) N_{1}+\varepsilon N_{2}\right) L \oplus L^{\prime}$ also spans $H$ over $\mathbf{Q}$. Hence, by replacing $N_{2}$ and $X$ again, we may assume that $\left((1-\varepsilon) N_{1}+\varepsilon N_{2}\right) L \oplus L^{\prime}$ spans $H$ over $\mathbf{Q}$ for any $0 \leq \varepsilon \leq 1$. By replacing $L$ by $L \oplus L^{\prime}$ and $N_{j}$ by $N_{j} \oplus$ id $(j=1,2)$, we may and will assume that $(1-\varepsilon) N_{1}+\varepsilon N_{2}$ is bijective for any $\varepsilon$, as desired.

Take a basis $\left(l_{j}\right)$ of $L$. For each $j$, consider an interval $I_{j} \subset \mathbf{R}$ of the form $(a, a+1]$ or $[a, a+1)$, where $a$ is an integer depending on the index $j$. Let $C$ be the subset of $X$ consisting of the elements of the form $\left(a_{1}, a_{2},\left(a_{1} N_{1}+a_{2} N_{2}\right)\left(\sum c_{j} l_{j}\right)\right)$, where $a_{1}, a_{2} \in \mathbf{Q} \geq 0$, and $c_{j} \in \mathbf{Q}$ belongs to $I_{j}$.

The given cone $\sigma$ is not necessarily contained in one of such a subset $C$, but, after further replacing $N_{2}$ and $X$ (as in the first paragraph of this proof), we may assume that it is the case, that is, there are $I_{j}$ of the above form such that the associated $C$ contains $\sigma$. Then, for any rational number $\varepsilon$ with $0 \leq \varepsilon \leq 1$, there are unique rational numbers $c_{\varepsilon, j}$ such that $(1-$ $\left.\varepsilon, \varepsilon,\left((1-\varepsilon) N_{1}+\varepsilon N_{2}\right)\left(\sum c_{\varepsilon, j} l_{j}\right)\right)$ belongs to $\sigma$. Note that $c_{\varepsilon, j}$ belongs to $I_{j}$. We may assume further that

$(*)$ the subset $\left\{j \mid c_{0, j}\right.$ is an integer $\}$ of indices contains $\left\{j \mid c_{\varepsilon, j}\right.$ is an integer $\}$ for any positive $\varepsilon \leq 1$. 
We prove that the desired condition is now satisfied with $\varepsilon_{0}=1$ and $\sigma^{\prime}=\sigma$. Assume that $(1,0,0)$ is not in $l(\sigma)$. It is enough to show that $(1-\varepsilon, \varepsilon, 0)$ is also not in $l(\sigma)$ for any positive rational number $\varepsilon \leq 1$. Let $c_{\varepsilon, j}$ be the rational numbers such that $\left(1-\varepsilon, \varepsilon,\left((1-\varepsilon) N_{1}+\varepsilon N_{2}\right)\left(\sum c_{\varepsilon, j} l_{j}\right)\right)$ belongs to $l(\sigma)$. Note that $c_{\varepsilon, j}$ belongs to some fixed translation of $I_{j}$. Then, these numbers satisfy the same condition $(*)$ as above. By assumption, $N_{1}\left(\sum c_{0, j} l_{j}\right)$ is not zero, so that, at least one of $c_{0, j}$ is not zero. By $(*)$ and the fact that $I_{j}$ contains only one integer, this implies that $c_{\varepsilon, j}$ is also not zero, and $\left((1-\varepsilon) N_{1}+\varepsilon N_{2}\right)\left(\sum c_{\varepsilon, j} l_{j}\right)$ is not zero for any positive $\varepsilon \leq 1$. This means that $l(\sigma)$ has the trivial intersection with $\mathbf{Q}_{>0}^{2} \times\{0\}$.

(2) Let $A:=N_{1} J+N_{2} J$. Then, the action of $L$ on $X$ induces the action of $L /(J \cap L)$ on

$$
\bar{X}:=\mathbf{Q}_{\geq 0}^{2} \times(H / A)
$$

because we have $a_{1} N_{1} l+a_{2} N_{2} l \in A$ for $a_{1}, a_{2} \in \mathbf{Q}_{\geq 0}$ if $l \in J$. Further, we have the operators

$$
\overline{N_{j}}: L /(J \cap L) \rightarrow H / A
$$

induced by $N_{j}$ for $j=1,2$.

Let $\bar{\sigma}$ be the image of $\sigma$ in $\bar{X}$, and we are in the situation in 2.1 with $H / A, \bar{X}, \bar{\sigma}, L /(J \cap L), \overline{N_{1}}$, and $\overline{N_{2}}$ for $H, X, \sigma, L, N_{1}$, and $N_{2}$ there.

We prove that $\overline{N_{1}}$ is injective. Let $l \in L$ with $N_{1}(l) \in A$. Since $A=$ $N_{1} J+N_{2} J$, there are $j_{1}, j_{2} \in J$ such that $N_{1}(l)=N_{1}\left(j_{1}\right)+N_{2}\left(j_{2}\right)$. From this, $N_{1}\left(l-j_{1}\right) \in N_{2} J$, so $l-j_{1} \in N_{1}^{-1} N_{2} J \subset J$ by the assumption. Hence $l \in J$, and $\overline{N_{1}}$ is injective.

Therefore, by (1), there is a positive $\varepsilon_{0} \leq 1$ such that any positive rational number $\varepsilon \leq \varepsilon_{0}$ satisfies the following condition: Let

$$
\overline{\sigma^{\prime}}=\bar{\sigma} \cap\left((H / A)_{1}+(H / A)_{1+\varepsilon}\right) .
$$

Then, for any $l \in L$, we have either $(1,0, \overline{0}) \in l\left(\overline{\sigma^{\prime}}\right)$ or $l\left(\overline{\sigma^{\prime}}\right) \cap\left(\mathbf{Q}_{\geq 0}^{2} \times\{\overline{0}\}\right)=$ $\{(0,0, \overline{0})\}$. Here and hereafter we denote by $\bar{h}$ the image of an element $h$ of $H$ in $H / A$.

We prove that this $\varepsilon_{0}$ satisfies the desired condition. To see this, fix any positive rational number $\varepsilon \leq \varepsilon_{0}$, and let 


$$
\sigma^{\prime}=\sigma \cap\left(H_{1}+H_{1+\varepsilon}\right),
$$

whose image in $\bar{X}$ is $\overline{\sigma^{\prime}}$. Let $l \in L$. Assume that $(1,0, \overline{0}) \in l\left(\overline{\sigma^{\prime}}\right)$. Then, $(1,0, h) \in l\left(\sigma^{\prime}\right)(h \in H)$ implies $(1,0, \bar{h}) \in l\left(\overline{\sigma^{\prime}}\right)$, and $\bar{h}=\overline{0}$, which means $h \in A=N_{1} J+N_{2} J$. Otherwise, $l\left(\overline{\sigma^{\prime}}\right) \cap\left(\mathbf{Q}_{\geq 0}^{2} \times\{\overline{0}\}\right)=\{(0,0, \overline{0})\}$, which contains the image of $l\left(\sigma^{\prime}\right) \cap\left(\mathbf{Q}_{\geq 0}^{2} \times\{0\}\right)$ in $\bar{X}$. Hence, the image is $\{(0,0, \overline{0})\}$. On the other hand, by Lemma 2.2 applied to $l\left(\sigma^{\prime}\right)$ as $\sigma$ there $\left(H_{1}+H_{1+\varepsilon}\right.$ as $X$ there $), l\left(\sigma^{\prime}\right)$ has the trivial intersection with the subset $\{(0,0)\} \times H$. Hence, $l\left(\sigma^{\prime}\right) \cap\left(\mathbf{Q}_{\geq 0}^{2} \times\{0\}\right)$ is trivial.

\section{Polarized nilpotent orbits}

One of the key facts which we will use later in the proof of the main theorem (Theorem 1.13) is the following proposition (Proposition 3.2) on a pure nilpotent orbit.

3.1 Let $H_{\mathbf{Z}}^{\prime}$ be a free $\mathbf{Z}$-module of finite rank, let $w$ be an integer, and let $\langle$,$\rangle be a non-degenerate (-1)^{w}$-symmetric pairing on $H_{\mathbf{Z}}^{\prime}$. Let $\left(h^{p, q}\right)_{p+q=w}$ be non-negative integers satisfying $h^{p, q}=h^{q, p}$ and $\sum h^{p, q}=\operatorname{rank}_{\mathbf{Z}} H_{\mathbf{Z}}^{\prime}$. Let $D$ be the associated Griffiths domain.

Let $G_{\mathbf{Q}}^{\prime}$ be the group of the $\mathbf{Q}$-automorphisms of $\left(H_{\mathbf{Q}}^{\prime},\langle\rangle,\right)$, and $\mathfrak{g}_{\mathbf{Q}}^{\prime}$ the associated Lie algebra.

Let $N_{1}, N_{2} \in \mathfrak{g}_{\mathbf{Q}}^{\prime} \subset \operatorname{End}_{\mathbf{Q}}\left(H_{\mathbf{Q}}^{\prime}\right)$ be mutually commutative nilpotent elements. Let $F \in \check{D}$. Assume that $\left(N_{1}, N_{2}, F\right)$ generates a nilpotent orbit $([9,5.4 .1])$.

Note that, in applying the results in this section to the main theorem in Section 4, we will use only the case $w=-1$.

We assume that the associated weight filtrations $W\left(N_{1}+N_{2}\right)[-w]$ and $W\left(N_{2}\right)[-w]$ coincide. We denote by $M$ this filtration.

Proposition 3.2 For any $n \geq 0$, we have

$$
M_{w-1} \cap \bigcap_{j=0}^{\infty}\left(M_{w-2}+\left(N_{2}^{j}\right)^{-1}\left(\operatorname{Im} N_{1}^{j+1}\right)\right) \cap\left(N_{2} N_{1}^{-1}\right)^{n}\left(M_{w-2}\right) \subset M_{w-2} .
$$

This is [10, Proposition 3.2]. See [10, 3.3-14] for the proof.

Proposition 3.3 Let the assumption be the same as above. Let $a_{1}, a_{2}$ be positive rational numbers, and let $N_{2}^{\prime}:=a_{1} N_{1}+a_{2} N_{2}$. 
Consider the increasing sequence $N_{1}^{-1} N_{2}(0), \quad\left(N_{1}^{-1} N_{2}\right)^{2}(0)$, $\left(N_{1}^{-1} N_{2}\right)^{3}(0), \ldots$ of subspaces of $H_{\mathbf{Q}}^{\prime}$, which is eventually stable, and let $J=\left(N_{1}^{-1} N_{2}\right)^{k}(0)(k \gg 0)$ be the stable subspace of $H_{\mathbf{Q}}^{\prime}$. Let $A=N_{2} J$. Similarly, let $J^{\prime}=\left(N_{1}^{-1} N_{2}^{\prime}\right)^{k}(0)(k \gg 0)$ and $A^{\prime}=N_{2}^{\prime} J^{\prime}$. Then we have $J=J^{\prime}$ and $A=A^{\prime}$.

These two equalities are (3) and (4) in the proof of [10, Proposition $3.22]$, and proved there.

\section{Proofs of main results}

Here we prove Theorem 1.13.

Convention Below, we adopt the following general convention: For any element $N \in \mathfrak{g}_{\mathbf{Q}}$, we denote its image in $\mathfrak{g}_{\mathbf{Q}}^{\prime}$ with the prime: $N^{\prime}$.

4.1 Let $\Gamma_{u}$ be the kernel of the natural projection $\Gamma \rightarrow \Gamma^{\prime}$, which is naturally isomorphic to the unipotent radical $G_{\mathbf{Z}, u}$ (cf. $[7,5.1 .7]$ ), and also naturally isomorphic to the additive group $H_{\mathbf{Z}}^{\prime}$, via the correspondence $\Gamma_{u} \ni$ $\gamma \leftrightarrow \gamma(e) \in H_{\mathbf{Z}}^{\prime}$. Here $e$ is the standard generator $1 \in \mathbf{Z} \subset H_{\mathbf{Z}}^{\prime} \oplus \mathbf{Z}=H_{\mathbf{Z}}$. Then, $\Gamma$ is isomorphic to a semi-direct product of $\Gamma_{u}$ and $\Gamma^{\prime}$.

4.2 First we claim that, in the statement of Theorem 1.13, we can replace " $\Gamma$ " with " $\Gamma_{u}$ ".

We prove this claim till the end of this subsection (Subsection 4.2). Below, we denote the image of an element $N \in \sigma\left(\subset \sigma^{\prime} \times_{\mathfrak{g}_{\mathbf{Q}}^{\prime}} \mathfrak{g}_{\mathbf{Q}}\right)$ in $\mathfrak{g}_{\mathbf{Q}}$ by the same symbol, and the image of an element $N^{\prime} \in \sigma^{\prime}$ in $\mathfrak{g}_{\mathbf{Q}}^{\prime}$ by the same symbol by abuse of notation.

Let $N_{1}^{\prime}, \ldots, N_{m}^{\prime}$ be a set of generators of the fs monoid $\sigma^{\prime} \cap \Gamma^{\prime}$. For each $j$ with $1 \leq j \leq m$, take the point $N_{j}$ of $\sigma$ whose image in $\sigma^{\prime}$ is $N_{j}^{\prime}$. Let $h_{j}:=N_{j}(e)$.

Let $\sigma^{\prime} \cap \Gamma^{\prime}$ act on $H_{\mathbf{Q}}^{\prime}$ through the map $\Gamma^{\prime} \rightarrow \mathfrak{g}_{\mathbf{Q}}^{\prime} \subset \operatorname{End}_{\mathbf{Q}}\left(H_{\mathbf{Q}}^{\prime}\right)$, which is induced by $a$ in 1.4 .

Since $N_{j}^{\prime}$ is nilpotent, the set

$$
S:=\left\{\frac{N_{j_{1}}^{\prime} \cdots N_{j_{k-1}}^{\prime}\left(h_{j_{k}}\right)}{k !} \mid k \geq 1,1 \leq j_{1}, \ldots, j_{k} \leq m\right\}
$$

is finite, and there is an integer $M>0$ such that the lattice $(1 / M) H_{\mathbf{Z}}^{\prime}$ 
contains this finite set:

(*) $\quad S \subset(1 / M) H_{\mathbf{Z}}^{\prime}$.

If we replace the lattice $H_{\mathbf{Z}}^{\prime}$ with $(1 / M) H_{\mathbf{Z}}^{\prime}$, the groups $G_{\mathbf{Z}}^{\prime}, G_{\mathbf{Z}}$, and hence $\Gamma$ become larger.

To prove the claim, we assume that the $\Gamma_{u}$-version of Theorem 1.13 holds. In particular, we assume that the $\Gamma_{u}$-version of Theorem 1.13 for $(1 / M) H_{\mathbf{Z}}^{\prime}$ holds. Note that this $\Gamma_{u}$ for $(1 / M) H_{\mathbf{Z}}^{\prime}$ is larger than the original one.

Hence, to see our claim, it is enough to prove the following (1) and (2) under this assumption because a subset of a weak fan is a weak fan if it is closed under the operation of taking a face.

(1) The action on $\sigma$ of any element of $\Gamma^{\prime}$ coincides with that of some element of the larger $\Gamma_{u}$.

(2) The action on $\sigma_{0}^{\prime}$ of any element of $\Gamma^{\prime}$ coincides with that of some element of the larger $\Gamma_{u}$.

Since (2) is the special case of (1), we prove only (1).

We see that, for any element $N$ of $\sigma$, there are non-negative rational numbers $a_{j}(1 \leq j \leq m)$ such that $N^{\prime}=\sum a_{j} N_{j}^{\prime}$ and that $N(e)=\sum a_{j} h_{j}$. In fact, take any $a_{j}$ such that $N^{\prime}=\sum a_{j} N_{j}^{\prime}$. We prove $N(e)=\sum a_{j} h_{j}$. Consider the element $\sum a_{j} N_{j}$. This is in $\sigma$ and maps to $N^{\prime}$ so that it coincides with $N$. Hence $N(e)=\sum a_{j} N_{j}(e)=\sum a_{j} h_{j}$.

Then the action of any element $\exp \left(L^{\prime}\right)\left(L^{\prime}=\sum m_{l} N_{l}^{\prime}, m_{l} \in \mathbf{Z}\right)$ of $\Gamma^{\prime}$ on the $H_{\mathbf{Q}^{-}}^{\prime}$-component of $N$ is described as

$$
\begin{aligned}
\sum a_{j} h_{j} & \mapsto \sum a_{j} h_{j}+\left(\exp \left(L^{\prime}\right)-1\right)\left(\sum a_{j} h_{j}\right) \\
& =\sum a_{j} h_{j}+\sum_{k \geq 1} \frac{L^{\prime k-1}}{k !} L^{\prime}\left(\sum a_{j} h_{j}\right) .
\end{aligned}
$$

But, we have

$$
\begin{aligned}
L^{\prime}\left(\sum_{j} a_{j} h_{j}\right) & =\left(\sum_{l} m_{l} N_{l}^{\prime}\right)\left(\sum_{j} a_{j} h_{j}\right) \\
& =\sum_{j, l} a_{j} m_{l}\left(N_{l}^{\prime}\left(h_{j}\right)\right)
\end{aligned}
$$




$$
\begin{aligned}
& =\sum_{j, l} a_{j} m_{l}\left(N_{j}^{\prime}\left(h_{l}\right)\right) \quad\left(\text { by } N_{l} N_{j}=N_{j} N_{l}\right) \\
& =\left(\sum_{j} a_{j} N_{j}^{\prime}\right)\left(\sum_{l} m_{l} h_{l}\right) .
\end{aligned}
$$

Hence the action is

$$
\sum a_{j} h_{j} \mapsto \sum a_{j} h_{j}+\left(\sum_{j} a_{j} N_{j}^{\prime}\right) \sum_{k \geq 1} \frac{L^{\prime k-1}}{k !}\left(\sum_{l} m_{l} h_{l}\right) .
$$

Since $\sum_{k>1}\left(L^{\prime k-1} / k !\right)\left(\sum_{l} m_{l} h_{l}\right)$ is in $(1 / M) H_{\mathbf{Z}}^{\prime}$ by $(*)$, this action is certainly realized by that of the corresponding element of the larger $\Gamma_{u}$, which completes the proof of (1).

4.3 In the rest of this section, we prove the $\Gamma_{u}$-version of Theorem 1.13. To prove it, we can replace $\sigma^{\prime}$ by each member of a finite subdivision of $\sigma^{\prime}$ and replace $\sigma$ by the inverse image of the member. Further, if such a member is of one dimension, it causes no problems (cf. Proposition 1.10). Hence, in the replacement, it is enough to consider only the members of 2-dimension.

Take a set of generators $N_{1}^{\prime}, N_{2}^{\prime}$ of $\sigma^{\prime}$. In the following, let $H_{\mathbf{Z}}^{\prime}$ act on $\mathfrak{g}_{\mathbf{Q}}$ via the isomorphism $H_{\mathbf{Z}}^{\prime} \cong \Gamma_{u}$ in 4.1 .

Consider the sequence of subspaces $J_{j}$ of $H_{\mathbf{Q}}$ defined as: $J_{0}=\{0\}$, $J_{j+1}=N_{1}^{\prime-1} N_{2}^{\prime} J_{j}(j \geq 0)$. Since the correspondence $X \mapsto N_{1}^{\prime-1} N_{2}^{\prime} X$ ( $X \subset H_{\mathbf{Q}}$ ) is order-preserving, the sequence $J_{j}$ is increasing, and eventually stable. Let $J$ be the stable subspace, that is,

$$
J:=\left(N_{1}^{\prime-1} N_{2}^{\prime}\right)^{k}(0)
$$

for a sufficiently large $k$. Let

$$
A:=N_{2}^{\prime} J
$$

Recall that $e$ is the standard generator 1 of $\mathbf{Q}$ of weight 0 . Recall also that $\sigma_{0}^{\prime}$ denotes the 0 -lift of $\sigma^{\prime}$.

First we prove that we may assume

(1) $W\left(N_{1}^{\prime}+N_{2}^{\prime}\right)=W\left(N_{2}^{\prime}\right)$ and for any $\gamma \in \Gamma_{u}$, we have either $N_{1}(e) \in A$ 
or $\operatorname{Ad}(\gamma)(\sigma) \cap \sigma_{0}^{\prime}=\{0\}$, where $N_{1}$ is the unique element of $\operatorname{Ad}(\gamma)(\sigma)$ lying over $N_{1}^{\prime}$ via the projection $\mathfrak{g}_{\mathbf{Q}} \rightarrow \mathfrak{g}_{\mathbf{Q}}^{\prime}$.

To see that we may assume (1), by the compactness argument as in [10, 6.6], it is enough to show (1) after replacing $N_{2}^{\prime}$ by $(1-\varepsilon) N_{1}^{\prime}+\varepsilon N_{2}^{\prime}$ for any sufficiently small $\varepsilon>0$, that is, it is enough for us to work around $N_{1}^{\prime}$ because the set $\left\{(1-a) N_{1}^{\prime}+a N_{2}^{\prime} \mid a \in \mathbf{Q}, 0 \leq a \leq 1\right\}$ is closed and bounded.

We may assume that $W\left(N_{1}^{\prime}+N_{2}^{\prime}\right)=W\left(N_{2}^{\prime}\right)$ by replacing $N_{2}^{\prime}$ by $(1 / 2) N_{1}^{\prime}+(1 / 2) N_{2}^{\prime}$ if necessary. Next, we want to apply Lemma $2.3(2)$. To do so, take $H_{\mathbf{Q}}^{\prime}, H_{\mathbf{Z}}^{\prime}, N_{1}^{\prime}, N_{2}^{\prime}$ as $H, L, N_{1}, N_{2}$ in 2.1. Identify $\sigma^{\prime}$ with $\mathbf{Q}_{\geq 0}^{2}$ by the correspondence $N_{1}^{\prime} \leftrightarrow(1,0), N_{2}^{\prime} \leftrightarrow(0,1)$. Then, the pullback of $\sigma^{\prime}$ by the projection $\mathfrak{g}_{\mathbf{Q}} \rightarrow \mathfrak{g}_{\mathbf{Q}}^{\prime}$, which is isomorphic to $\sigma^{\prime} \times H_{\mathbf{Q}}^{\prime}$, is identified with $X$ in 2.1. Take $J$ in the above as the subspace $J$ in Lemma 2.3. Then clearly $N_{1}^{\prime-1} N_{2}^{\prime} J=J$. Apply Lemma $2.3(2)$, and, since we have $N_{1}^{\prime} J+N_{2}^{\prime} J=N_{2}^{\prime} J=A$, we see (1) after replacing $N_{2}^{\prime}$, as desired.

4.4 Hereafter we assume (1) in 4.3. We will prove that the set

$$
\begin{aligned}
& \left\{\operatorname{Ad}(\gamma)(\tau) \mid \gamma \in \Gamma_{u}, \tau \text { is a face of } \sigma\right\} \\
& \cup\left\{\operatorname{Ad}(\gamma)\left(\tau_{0}^{\prime}\right) \mid \gamma \in \Gamma_{u}, \tau \text { is a face of } \sigma\right\}
\end{aligned}
$$

is a weak fan.

Let $\sigma_{1}, \sigma_{2}$ be two elements of this set. It suffices to show that the condition 1.7 (2) is satisfied. Since it is trivial if one of them is 0 or 1dimensional, we may assume that both are 2-dimensional.

First, suppose $\sigma_{1}=\operatorname{Ad}(\gamma)\left(\sigma_{0}^{\prime}\right)$ for some $\gamma \in \Gamma_{u}$ and $\sigma_{2}=\sigma_{0}^{\prime}$. Then, the condition 1.7 (2) is satisfied by [7, Theorem 6.2.1]. The proof of [7, Theorem 6.2.1] also works for $\sigma_{1}=\operatorname{Ad}(\gamma)(\sigma)$ for some $\gamma \in \Gamma_{u}$ and $\sigma_{2}=\sigma$ (see [8, Theorem 3.1]).

Hence the rest is to show $1.7(2)$ for $\sigma_{1}=\operatorname{Ad}(\gamma)(\sigma)$ for some $\gamma \in \Gamma_{u}$ and $\sigma_{2}=\sigma_{0}^{\prime}$. We may assume that $\gamma=1$. If $\sigma \cap \sigma_{0}^{\prime}=\{0\}$, then 1.7 (2) is clearly satisfied. Hence, by (1) in 4.3, what to see is the following (2), which completes the proof of Theorem 1.13.

(2) Let $F \in \check{D}$. Assume that $\left(\sigma_{\mathfrak{g}}, F\right)$ and $\left(\left(\sigma_{0}^{\prime}\right)_{\mathfrak{g}}, F\right)$ generate nilpotent orbits, and that $\sigma$ and $\sigma_{0}^{\prime}$ have a common interior point. Assume also that $N_{1}(e) \in A$, where $N_{1}$ is the unique element of $\sigma$ lying over $N_{1}^{\prime}$. Then, $\sigma$ coincides with $\sigma_{0}^{\prime}$. 
4.5 Let the notation and the assumption be as in 4.4 (2). We prove $N_{1}(e) \in M_{-3}$, where $M=M\left(N_{1}+N_{2}, W\right)$. First, the admissibility implies

(3) $N_{1}(e) \in \operatorname{Im}\left(N_{1}^{\prime}\right)$.

In fact, take an element $e+h\left(h \in H_{\mathbf{Q}}^{\prime}\right)$ of $M_{0}$. Then, $N_{1}(e)+N_{1}(h) \in$ $M_{-2} \cap W_{-1}=\left(W\left(N^{\prime}\right)[1]\right)_{-2} \subset \operatorname{Im}\left(N_{1}^{\prime}\right)$. Hence, (3) follows.

Next, since $\sigma$ and $\sigma_{0}^{\prime}$ have a common interior point, there is a positive rational number $\varepsilon<1$ such that $(1-\varepsilon) N_{1}+\varepsilon N_{2}$ is in $\sigma_{0}^{\prime}$. Then,

(4) $\left((1-\varepsilon) N_{1}+\varepsilon N_{2}\right)(e)=0$.

Since $e \in W_{0}$ and $M=M\left((1-\varepsilon) N_{1}+\varepsilon N_{2}, W\right)$, by the well-known property of the relative weight filtration (see, for example, [7, 1.2.1.3]), (4) implies that

(5) $e \in M_{0}$.

Since $N_{1}$ sends $M_{w}$ into $M_{w-2}$ for any $w$, we have

(6) $N_{1}(e) \in M_{-2}$.

Next, since $\sigma$ is a nilpotent cone, we have $N_{1} N_{2}=N_{2} N_{1}$. Hence, by $(4)$,

$$
\begin{aligned}
0 & =N_{1}\left((1-\varepsilon) N_{1}+\varepsilon N_{2}\right)(e) \\
& =\left((1-\varepsilon) N_{1}+\varepsilon N_{2}\right)\left(N_{1}(e)\right) \\
& =\left((1-\varepsilon) N_{1}^{\prime}+\varepsilon N_{2}^{\prime}\right)\left(N_{1}(e)\right) .
\end{aligned}
$$

Letting $N_{2}^{\prime \prime}:=(1-\varepsilon) N_{1}^{\prime}+\varepsilon N_{2}^{\prime}$, we have

(7) $N_{1}(e) \in \operatorname{Ker}\left(N_{2}^{\prime \prime}\right)$.

On the other hand, by Proposition 3.3,

(8) $N_{1}(e) \in A=\left(N_{2}^{\prime} N_{1}^{\prime-1}\right)^{k}(0)=\left(N_{2}^{\prime \prime} N_{1}^{\prime-1}\right)^{k}(0) \quad(k \gg 0)$.

Combining (3), (6), (7) and (8), we see $N_{1}(e) \in M_{-2} \cap \operatorname{Im}\left(N_{1}^{\prime}\right) \cap$ $\operatorname{Ker}\left(N_{2}^{\prime \prime}\right) \cap\left(N_{2}^{\prime \prime} N_{1}^{\prime-1}\right)^{k}(0)$.

Since $\operatorname{Ker}\left(N_{2}^{\prime \prime}\right) \subset \bigcap_{j=1}^{\infty}\left(\left(N_{2}^{\prime \prime j}\right)^{-1}\left(\operatorname{Im} N_{1}^{\prime j+1}\right)\right)$, by Proposition 3.2, we conclude

(9) $N_{1}(e) \in M_{-3}$. 
4.6 We continue to work under the assumption in (2) in 4.4. Next we prove that, using the Griffiths transversality, $N_{1}(e)$ is in $F^{-1} \cap \bar{F}^{-1}$. Since $F\left(\operatorname{gr}_{0}^{W}\right)^{0}=\mathbf{C}$, there is an element $h$ of $H_{\mathbf{C}}^{\prime}$ such that $e+h \in F^{0}$. Since $\left(\sigma_{\mathfrak{g}}, F\right)$ generates a nilpotent orbit, we have $N_{1}(e)+N_{1}^{\prime}(h) \in F^{-1}$. Similarly, since $\left(\left(\sigma_{0}^{\prime}\right)_{\mathfrak{g}}, F\right)$ generates a nilpotent orbit, we have $N_{1}^{\prime}(h) \in F^{-1}$. Hence, $N_{1}(e)=\left(N_{1}(e)+N_{1}^{\prime}(h)\right)-N_{1}^{\prime}(h) \in F^{-1}$. Since this element is real, it is also in $\bar{F}^{-1}$.

Together with $(9)$ in 4.5 , we see that $N_{1}(e)$ belongs to the subspace $F^{-1} \cap \bar{F}^{-1} \cap M_{-3}$, which vanishes by the fact that $(M, F)$ is a mixed Hodge structure. Hence, $N_{1}(e)=0$. Thus, two 2-dimensional cones $\sigma$ and $\sigma_{0}^{\prime}$ have a common 1-face. Since they also have a common interior point by assumption, they coincide, which completes the proof of (2) in 4.4 and Theorem 1.13 .

\section{Algebraicity of zero loci}

Here, as an application of Corollary 1.14, we give an alternative proof of the following theorem, which is a special case of a theorem by P. Brosnan and G. Pearlstein [1] (independently proved by C. Schnell [13] and by K. Kato, C. Nakayama, and S. Usui [6]).

Theorem 5.1 Let $S$ be a complex manifold. Let $Y$ be a divisor with normal crossings on $S$. Assume that for each point of $S$, there are at most two components passing through it. Let $H$ be a variation of PHS of weight -1 and let $J(H)$ be the associated intermediate Jacobian. Let $\nu: S^{*}:=$ $S-Y \rightarrow J(H)$ be an admissible normal function $([11])$. Let $Z(\nu) \subset S^{*}$ be its zero locus. Then, the closure of $Z(\nu)$ in $S$ is analytic.

Corollary 5.2 Let $S^{*}$ be a smooth complex algebraic surface. Let $H$ be a variation of PHS of weight -1 and let $\nu: S^{*} \rightarrow J(H)$ be an admissible normal function. Then the zero locus $Z(\nu) \subset S^{*}$ is algebraic.

5.3 To deduce Corollary 5.2 from Theorem 5.1, we take a smooth compactification $S$ of $S^{*}$ such that $Y:=S-S^{*}$ satisfies the assumption in Theorem 5.1. (Here, we correct a misprint in the part of [6] corresponding to this step: In the second last paragraph in Introduction of $[6], " k=0,1$ " should be " $k=0, w$, where $w$ is the weight of $H$ in $0.2 "$.

To prove Theorem 5.1, we endow $S$ with the log structure associated to 
$Y$.

Let $\nu_{0}: S^{*} \rightarrow J(H)$ be the zero section. Then, $Z(\nu)=\left\{s \in S^{*} \mid \nu(s)=\right.$ $\left.\nu_{0}(s)\right\}$. It is enough to prove that there is a closed analytic subset $Z$ such that $Z \cap S^{*}=Z(\nu)$.

Since the problem is local on $S$ and since we can replace $S$ by a finite ramified covering, we may and do assume that the local monodromy groups along $Y$ are unipotent. Then, $H$ extends uniquely to a log mixed Hodge structure on $S$, which is still denoted by $H$.

5.4 By Corollary 1.14, we may assume that there is a log Néron model $J_{S, \Sigma}$ for $\nu$ and $\nu_{0}$. We denote by $\tilde{\nu}$ and $\tilde{\nu}_{0}$ the extended sections, and let $Z:=\left\{s \in S \mid \tilde{\nu}(s)=\tilde{\nu}_{0}(s)\right\}$. We may assume that $S$ is Hausdorff. Then, $J_{S, \Sigma}$ is also Hausdorff so that $Z$ is a closed analytic subset by Proposition in [4, Section 4]. Since $Z(\nu)=Z \cap S^{*}$, this completes the proof of Theorem 5.1 .

Acknowledgments The author is thankful to K. Kato and S. Usui for collaboration on log intermediate Jacobians, from which this subject arose. The author thanks J. C. for suggesting this work. He also thanks the referee for the careful reading and useful comments. He was partially supported by JSPS Grants-in-Aid for Scientific Research (C) 22540011 and (B) 23340008 .

\section{References}

[ 1 ] Brosnan P. and Pearlstein G., On the algebraicity of the zero locus of an admissible normal function, Compositio Mathematica 149-11 (2013), 19131962.

[ 2 ] Kato K., Matsubara T. and Nakayama C., Log $C^{\infty}$-functions and degenerations of Hodge structures, Advanced Studies in Pure Mathematics 36, Algebraic Geometry 2000, Azumino (Ed. S. Usui, M. Green, L. Illusie, K. Kato, E. Looijenga, S. Mukai and S. Saito), Math. Soc. Japan, Tokyo, 2002, 269-320.

[3] Kato K., Nakayama C. and Usui S., Log intermediate Jacobians, Proc. Japan Academy 86-A-4 (2010), 73-78.

[ 4 ] Kato K., Nakayama C. and Usui S., Moduli of log mixed Hodge structures, Proc. Japan Academy 86-A-7 (2010), 107-112.

[ 5 ] Kato K., Nakayama C. and Usui S., Néron models in log mixed Hodge theory by weak fans, Proc. Japan Academy 86-A-8 (2010), 143-148. 
[6] Kato K., Nakayama C. and Usui S., Analyticity of the closures of some Hodge theoretic subspaces, Proc. Japan Academy 87-A-9 (2011), 167-172.

[ 7 ] Kato K., Nakayama C. and Usui S., Classifying spaces of degenerating mixed Hodge structures, III: Spaces of nilpotent orbits, Journal of Algebraic Geometry 22-4 (2013), 671-772.

[ 8 ] Kato K., Nakayama C. and Usui S., Néron models for admissible normal functions, Proc. Japan Academy 90-A-1 (2014), 6-10.

[9] Kato K. and Usui S., Classifying spaces of degenerating polarized Hodge structures, Ann. of Math. Stud., 169, Princeton Univ. Press, Princeton, NJ, 2009.

[10] Nakayama C., Log Néron models over surfaces, J. Math. Sci. Univ. Tokyo 19 (2012), 613-659.

[11] Saito M., Admissible normal functions, Journal of Algebraic Geometry 5-2 (1996), 235-276.

[12] Schmid W., Variation of Hodge structure: The singularities of the period mapping, Invent. Math. 22 (1973), 211-319.

[13] Schnell C., Complex analytic Néron models for arbitrary families of intermediate Jacobians, Invent. Math. 188 (2012), 1-81.

[14] Usui S., Variation of mixed Hodge structure arising from family of logarithmic deformations II: Classifying space, Duke Math. J. 51-4 (1984), 851-875.

Department of Economics

Hitotsubashi University

2-1 Naka, Kunitachi, Tokyo 186-8601

Japan

E-mail: c.nakayama@r.hit-u.ac.jp 